- Describes a case of delayed healing following root canal treatment.

- Discusses the importance of serial radiographs in monitoring and management of persistent periapical disease.

- Summarises expected healing timescale and processes.

\title{
Radiographic evidence of postoperative healing 12 years following root canal treatment - a case report
}

\author{
M. B. M. Thomas, ${ }^{1}$ S. J. Hayes ${ }^{2}$ and A. S. M. Gilmour ${ }^{3}$
}

A case with evidence of radiographic healing of a periapical lesion 12 years after root canal treatment is presented. The use and limitations of previously produced guidelines on radiographic follow-up intervals are discussed. Questions are raised about what is known of the healing process and when further treatment should be instituted. The reliance on single radiographs alone when making treatment decisions is discouraged.

\section{INTRODUCTION}

Apical periodontitis is a chronic inflammatory disorder of periradicular tissues caused by aetiological agents of endodontic origin. ${ }^{1}$ Following the thorough shaping, cleaning and obturation of the complex canal anatomy, healing can be expected in approximately $84 \%$ of cases. ${ }^{2}$ However, the post operative assessment of healing can be difficult and the diagnosis and management of persistent apical periodontitis is more variable among clinicians. ${ }^{3}$ Often the decision to re-treat is based on a number of independent factors, some of which are subjective. These include the presence of clinical signs and symptoms, a poorly condensed root filling, under filled and unfilled canals and persistent periapical radiographic changes. In addition,

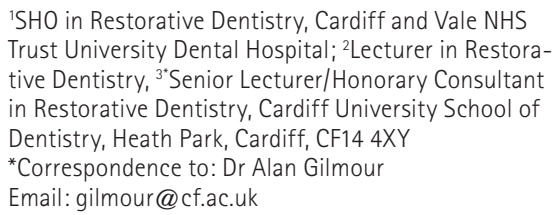

the intention to undertake other coronal procedures such as a crown or bridge may influence the decision process. Knowledge of the expected natural history of apical periodontitis, in particular healing times, will have bearing on the timing of interceptive measures. Persistent post-treatment disease is a common finding, even with stringent procedures, verified radiographically through epidemiological studies in $40-50 \%$ of root treated teeth. ${ }^{4}$ The severity of persistent disease will vary from mild asymptomatic to severe symptomatic pathology - should this have a bearing on treatment decisions? Therefore the questions that are asked post-operatively rely on patient symptoms, signs of infection and radiographic appearance (in particular repeat radiographs showing boney infill). Current literature would suggest that complete resolution of a periapical radiolucency should occur within four years in ideal circumstances. ${ }^{5}$ If this was not evident in a particular case, clinicians may view it suspiciously when planning further restorative intervention (especially fixed prosthodontics).

The aims and objectives of root canal treatment are well defined. Although materials and methods used for achieving our goals have altered radically over recent years, certain questions remain unanswered:

1. How long does post-operative healing take?

2. Is a persistent radiolucency the result of healing or of persistent or recurrent disease?

3. When is it appropriate to re-treat?

This paper describes an example of post-operative healing, evident on follow-up radiography, over a 12 year period after completion of root canal treatment. One of the issues dealt with

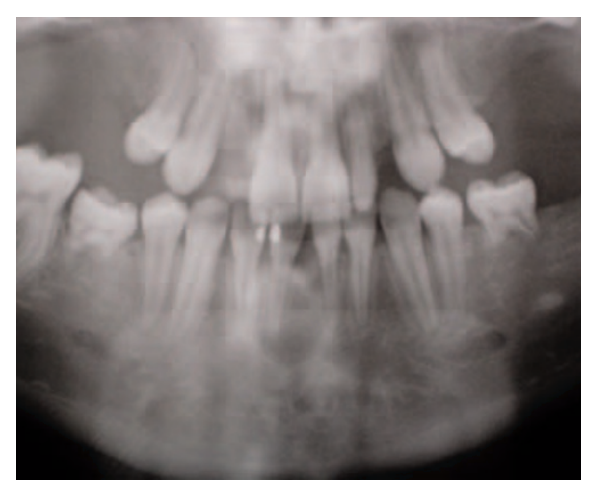

Fig. 1 OPT showing periapical radiolucency size at presentation at the apex of 41 


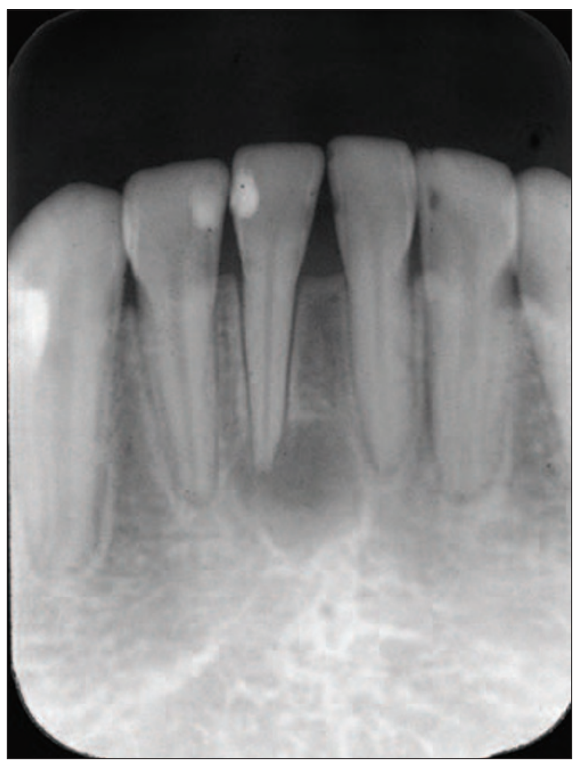

Fig. 2 Periapical radiograph on 21 October 1992 showing defined $7 \mathrm{~mm}$ diameter radiolucency associated with the 41

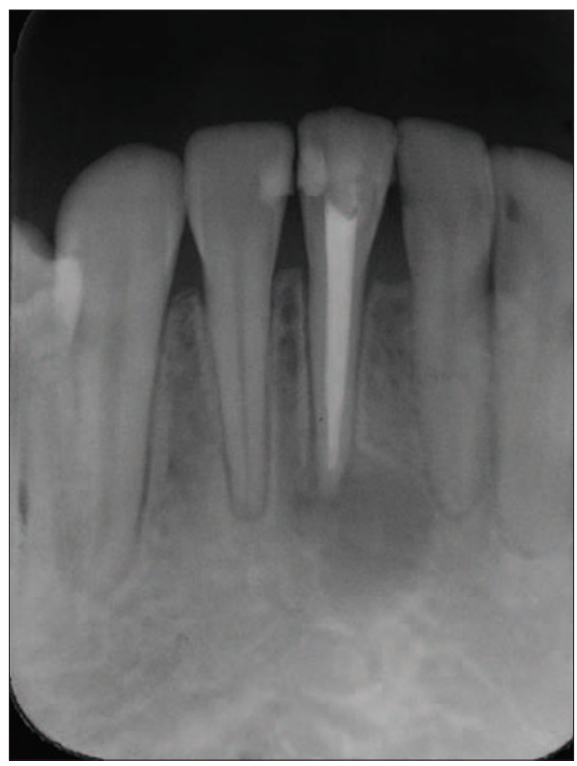

Fig. 4 Periapical radiograph taken upon completion of obturation in March 1994

in this paper is how this healing should be defined and the difficulties of clinical interpretation where previous radiographs are not available.

\section{CASE HISTORY}

The case of a 27-year-old female patient is presented. She was first seen at the Dental Hospital in Cardiff in 1990 suffering with severe hypodontia (missing a total of 16 teeth). She had been treated in a dental hospital setting for approximately two years with fixed appliance therapy. Her medical history was unremarkable. When seen on 21 July 1992 (age 13) a well defined radiolucency continuous with the periodontal ligament space was

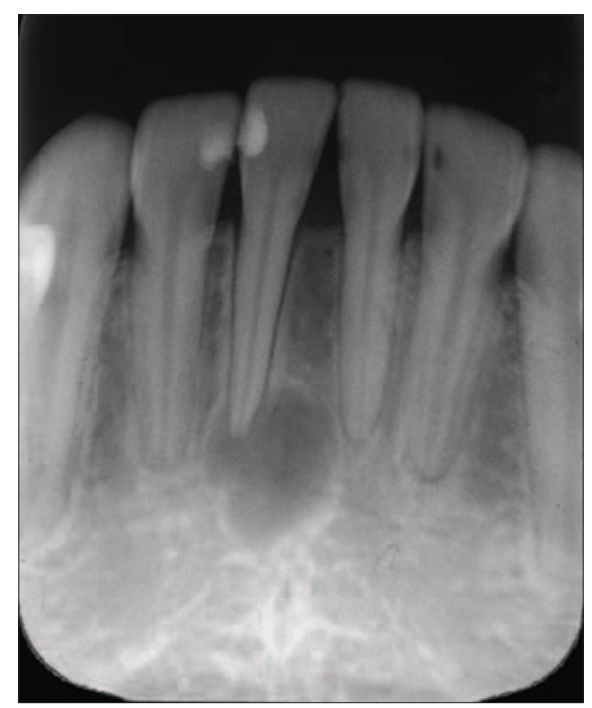

Fig. 3 Periapical radiograph taken 8 November 1993, approximately 14 months after initial presentation, showing a possible increase in the size of the radiolucency to $9 \mathrm{~mm}$, as compared to Figure 2

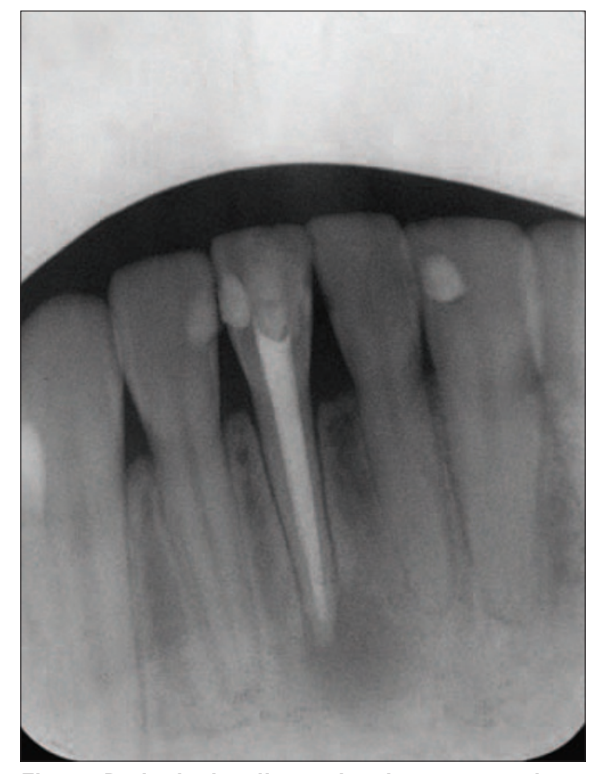

Fig. 5 Periapical radiograph taken 14 months after the RCT showing evidence of boney in-fill

noted associated with the 41 . This was an incidental finding following orthodontic de-bond and the patient was asymptomatic (Fig. 1). The patient was referred to the paediatric dentistry department where following clinical investigation and further radiographic investigation, root canal treatment was suggested to the patient and her parent on 21 October 1992 (Fig. 2). Initially the patient and parent declined treatment until early 1994 when extirpation of the pulp was commenced following referral to the restorative department. A preoperative radiograph suggested that the lesion had increased in size slightly from $7 \mathrm{~mm}$ at its widest point to $9 \mathrm{~mm}$ in the intervening 14 months
(Fig. 3). The lesion was well defined, oval in outline, partially corticated and homogenous in radiodensity. Root canal preparation was performed under rubber dam using a step-back approach. Obturation was completed with cold lateral condensation on the 3 March 1994 and the radiograph demonstrated a satisfactory root filling which was used as the baseline for monitoring post-operative healing (Fig. 4). During the course of root canal treatment the patient suffered from two episodes of severe pain and swelling and was prescribed amoxicillin.

At follow-up in 1995 approximately one year after RCT no symptoms were reported. Radiographic review confirmed the presence of an apical radiolucency although this was smaller than the baseline radiograph with some evidence of bony in-fill (Fig. 5). Review in the subsequent years - 1996 and 1997 - revealed no symptoms, however, radiographic examination showed the persistence of periapical rarefaction with little obvious change in size but curiously some changes in shape, with the maximum diameter of the lesion changing from the horizontal to the vertical plane (Figs 6a-b).

The patient was lost to follow-up until 2001 (some seven years after the RCT) when, still asymptomatic, further radiographic examination was undertaken. Figure 7 shows that there had been dramatic reduction in periapical radiolucency size around the 41 since the relatively static period from 1995 to 1997 . However, there remained a well defined $3 \mathrm{~mm}$ radiolucency continuous with the periodontal ligament space that would require further follow up.

At the most recent recall appointment in November 2006, 12 years after treatment, again no symptoms were reported and a further periapical radiograph was exposed (Fig. 8). Surprisingly this showed that there had been a further reduction in the size of the apical radiolucency and what remained was a well defined but slightly larger than normal apical periodontal ligament space.

The patient is currently in the middle of a course of restorative treatment and appropriate monitoring and review of 41 is planned in the future.

\section{DISCUSSION}

The diagnosis, management and monitoring of periapical pathology following 
root canal treatment requires thorough clinical and radiographic examination. The expected normal healing of the periapical tissue following RCT consists of complex regeneration of bone, cementum and periodontal ligament. ${ }^{6}$ This occurs at a histological level which clinicians are unable to monitor routinely. Therefore, the most common method of observing pathological alterations is via radiographic diagnosis.

The rarefaction seen radiographically is caused by replacement of the adjacent bone with inflammatory infiltrate since bone is less resistant than tooth substance to the resorptive products of inflammation such as cytokines and prostaglandins. Changes in the mineralization and structure of bone adjacent to the site of inflammation forms the basis of radiographic diagnostic procedures for the detection and monitoring of chronic apical periodontitis. ${ }^{6,7}$ The value of radiographs in the diagnosis of periapical disease is only realised once approximately $7 \%$ of mineral loss and loss of cortical plate has occurred. ${ }^{2}$ The width of the periodontal ligament space and integrity of lamina dura are considered important parameters in registering apical periodontitis. ${ }^{7}$

Radiographs are used to determine whether healing has occurred or whether a diagnosis of post treatment disease is appropriate. After RCT there may be a slight increase in the size of the radiolucency followed by in-fill of less organised bone proceeding from the outside to the centre and eventually the lamina dura and PDL return to normal. Healing of the periapical lesion usually occurs with hard tissue regeneration that is characterised by reduction of the radiolucency on follow-up radiographs. ${ }^{1}$ One unbiased method of reviewing systematic radiographs is the Periapical Index (PAI). The PAI is a scoring system of radiographic apical periodontitis that can be used not only for epidemiological and clinical trials but also in retrospective analysis of treatment results. ${ }^{7}$ The PAI allows assessment of periapical condition from radiographic images. The index is based on an ordinal scale made of five scores ranging from 1 (healthy) to 5 (severe periodontitis with exacerbating features) (Fig. 9). Ørstavik explains that a PAI of 3 on a root-treated tooth shows definite but not completely healed cases. The creators of the PAI point out

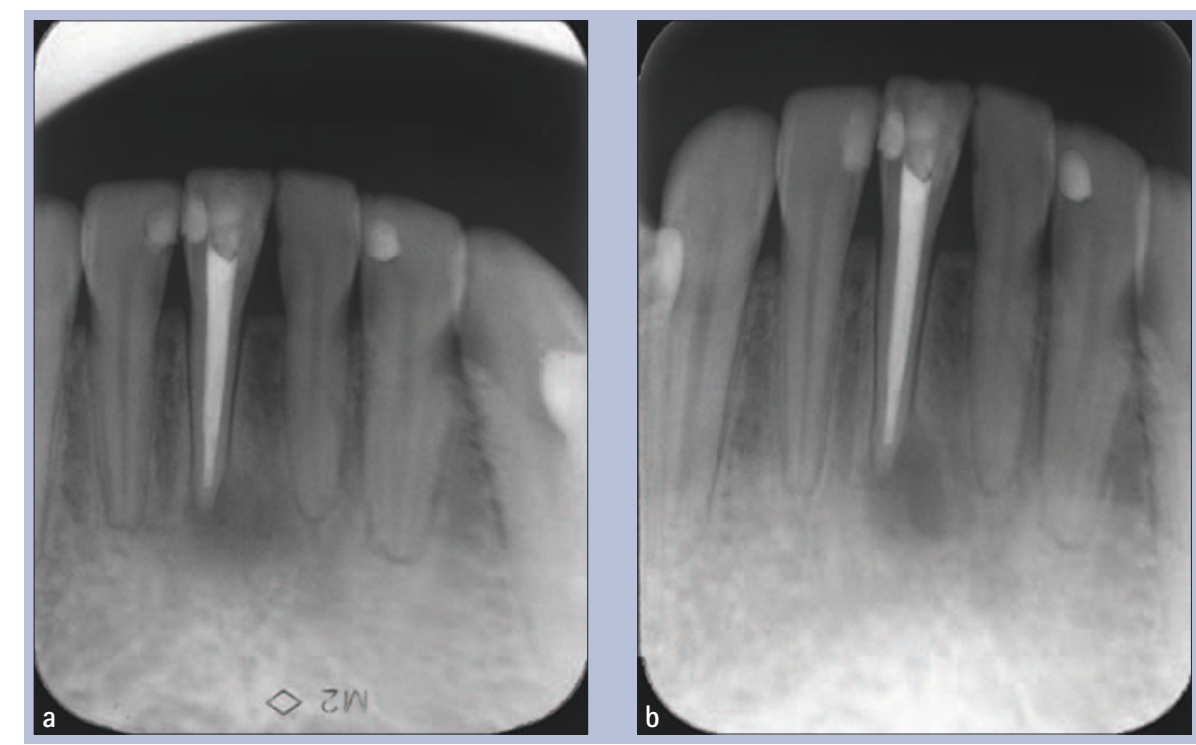

Figs 6a-b Sizes and shapes of apical radiolucencies in 1996 and 1997 respectively

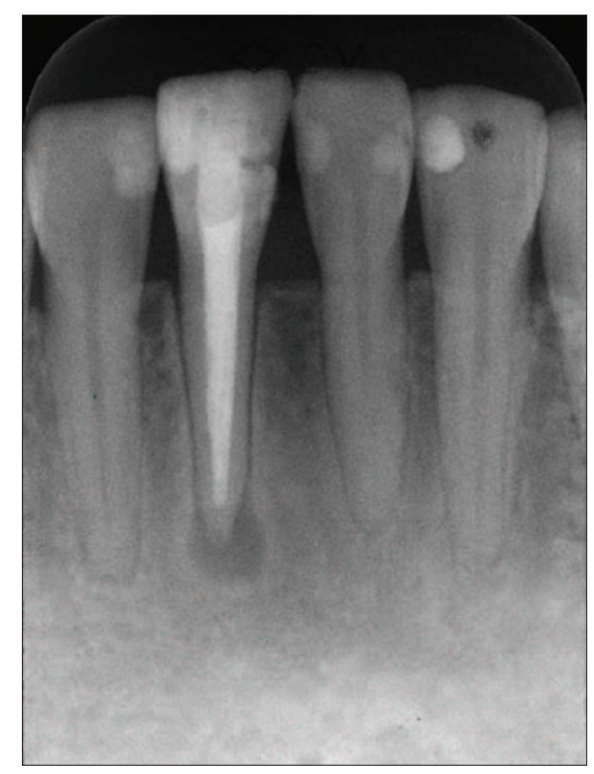

Fig. 7 Periapical radiograph taken in December 2001 showing obvious reduction in size of radiolucency since baseline March 1994

that the time a tooth will rest at a given score may vary widely among cases. They conclude that endodontic therapy produces a situation where periapical inflammatory lesions spend a long time at scores 2 and 3. In terms of success and failure Ørstavik and his colleagues suggest that a root filled tooth with PAI 4 or 5 should be deemed a definite failure, but would not consider a tooth for retreatment with a score of 3 and would deem such treated teeth as a success. ${ }^{7}$

Following orthograde RCT complete healing has been shown to take as long as four years to occur though healing is initiated in $89 \%$ of cases after one year. ${ }^{5}$ When complete healing does occur, it is diagnosed in $51 \%$ of cases after one

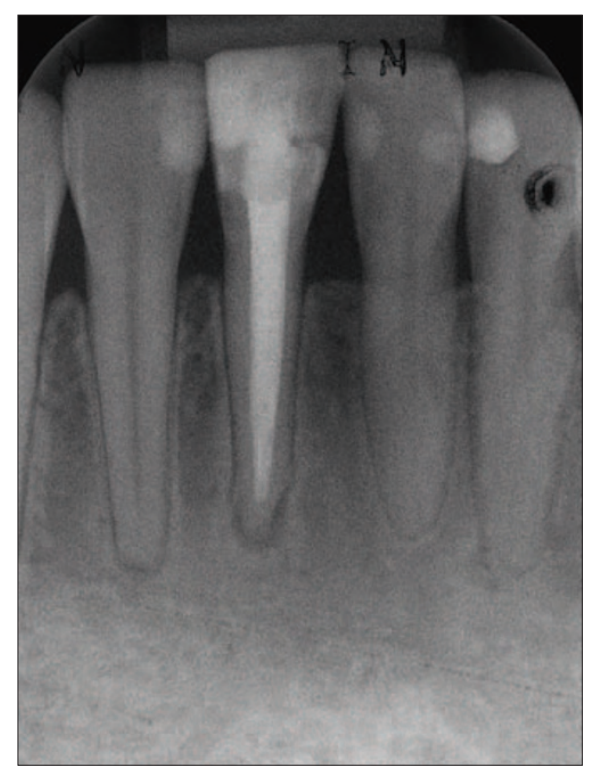

Fig. 8 Periapical radiograph taken in November 2006, 12 years following RCT, showing evidence of continued healing

year. ${ }^{5}$ However, in other studies healing was shown to take up to seven and even ten years to complete. ${ }^{6,8}$ Strindberg recommended a four year cut off period for radiographic follow-up of endodontically treated teeth. ${ }^{8}$ However, one published study found after ten to 17 years there were more late successes than failures and there was no definite observation period after which every case was stable. ${ }^{9}$

Clinical studies evaluating the success of root canal treatment often use radiographs as an outcome measure. Justification for re-treatment also often uses radiographic evidence. Radiographic recall has been advised annually for up to four years after RCT based on expected 


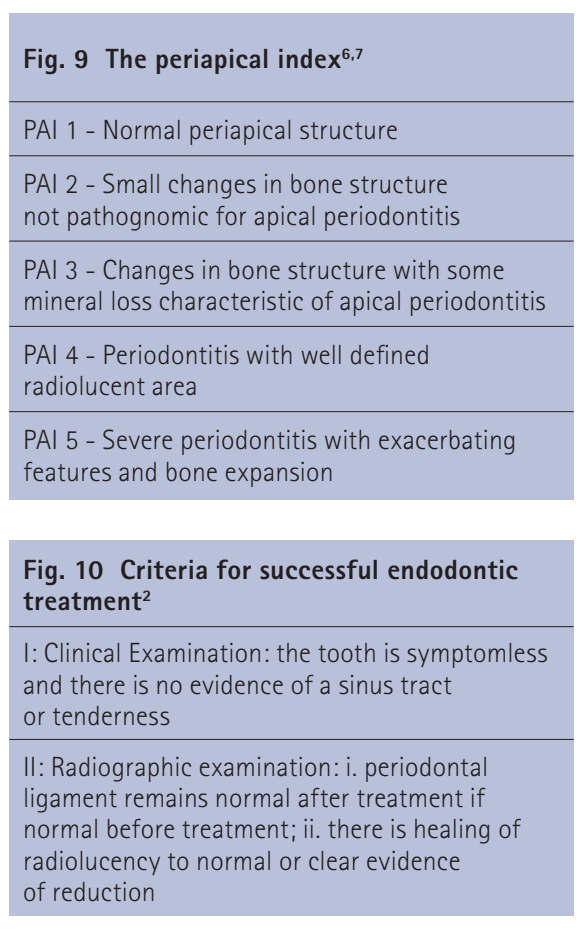

healing time, a time during which the healing curve flattens out. ${ }^{4,5}$ Guidelines have previously recommended that a follow up radiograph be taken at one year after treatment to assess healing, and if healing is incomplete, further annual radiographs are recommended until healing occurs. ${ }^{10}$ However, strictly followed guidelines may occasionally result in interceptive measures if healing is not seen as expected. This may mean that certain teeth are treated that would have healed if more time had been given.

The case presented in this paper is consistent with previous findings showing evidence of ongoing healing of more than four years, in this case 12 years following treatment. It can be justified therefore that in the face of no clinical symptoms and continued reduction of radiolucency size root filled teeth can be observed radiographically for more than four years. It follows that sufficient time must be given to allow for healing. If a period of only four years had been used in the case presented it is likely that further treatment may have been initiated unnecessarily. One significant dilemma occurs with the interpretation of a single radiograph prior to fixed prosthodontic work. This may reveal a periapical radiolucency that, without a set of serial radiographs showing a reducing lesion, may result in unnecessary re-treatment.

Smith et al. remind us that there is no clear definition of what constitutes success or failure in endodontic treatment. ${ }^{2}$
In terms of radiographic success, if there is a reduction in the size of a pre-operative periapical radiolucency then it may be deemed a success in the absence of clinical signs and symptoms. ${ }^{11}$ It has been suggested that the absence of clinical signs and symptoms irrespective of satisfactory radiographic features indicate success, while others state there must also be evidence of complete boney repair. ${ }^{8,12}$ There were no clinical signs or symptoms in the case presented after 1994 and clear evidence of a decreasing radiolucency, therefore based on the work by Byström et al. the treatment could be deemed successful. ${ }^{13}$

It must be realised that when deciding if a treatment is successful or not, this must depend on which definition of success is used. Definitions of success have previously been summarised elsewhere. ${ }^{14}$ Previous work has suggested that the term healing/disease should be used rather than success/failure. ${ }^{5,14}$ This defined healing as reduced radiolucency and clinical normality. Healed was defined as no clinical signs, symptoms or radiolucency. Finally, disease was defined as either persistent radiolucency or new radiolucency development with or without clinical signs. During the radiographic follow-up of this patient, judgements had to be made on whether the treatment had failed or not. There was an obvious, but small, decrease in the size of the lesion during the majority of the follow-up period and as previously reported provided there is a continuous decrease in the size of the lesion there is no need to judge a case a failure. ${ }^{2}$ However, it may have been justified in 1997, three years after obturation to consider the tooth for surgical treatment as a significant well defined radiolucency still remained. Using the PAI scoring system ${ }^{7}$ the case presented remained PAI 4 for some time and probably did not become a PAI 3 (deemed successful treatment) until seven years after completion of treatment and therefore, under certain circumstances, could have been re-treated if such guidelines were followed closely.

One study used the criterion for complete healing as that the radiographic width of the periodontal space was normal or slightly widened but less than 0.5 $\mathrm{mm} \cdot{ }^{13}$ In the case presented this width was only demonstrated in the most recent radiograph after 12 years (Fig. 8). Would it have been justified to deem this case a failure up until this point? Another study stated that if there was no or little reduction in radiolucency size after five years the treatment had failed. ${ }^{2}$ Based on the last set of criteria the case presented may have been deemed a failure up until the more recent radiographs. It has been shown that failures continue to occur for many years after completion of endodontic treatment and so there is continuing requirement for patient recall and no period after which failure is unlikely. ${ }^{2}$ It is clear to see that opinion on the definition of healing or success is varied. A useful set of criteria for successful treatment is shown in Figure 10.

It has been concluded that radiographic techniques alone lack sufficient sensitivity to serve as a reliable means for diagnosing periapical health. ${ }^{4}$ If we rely on single 'snapshot' radiographs alone we may be caught out by diagnosing disease in a healing case or vice versa; this will impact on treatment decisions. The benefit of serial radiographs in the diagnosis of healing as opposed to active lesions cannot be overemphasised. Attitudes to periapical disease vary among individuals and this affects the decision making process. Reit suggests there are two re-treatment decision making principles. The first principle is to treat any tooth with a periapical lesion that is not expected to heal. If the first principle is not followed reference must be made as to the reason ie to respect patient autonomy, re-treatment risks or monetary cost. ${ }^{15}$ The patient in this case did not return for several years during followup which influenced decision making. If options other than monitoring were chosen this patient may have been treated unnecessarily.

\section{CONCLUSION}

There would appear to be disagreement on how long periapical healing takes. This case demonstrates delayed healing, compared to accepted healing timescale, which could have resulted in further intervention based on the radiographic appearance alone. We would suggest that where previous radiographs are not available the risk of intervention in the form of re-treatment is increased. The clinician should be aware of the possibility of ongoing delayed healing and where possible use serial radiographs to inform the decision making process. 
1. Nair PN R. On the causes of persistent apical periodontitis: a review. Int Endod J 2006; 39: 249-281.

2. Smith C S, Setchell D J, Harty F J. Factors influencing the success rate of conventional root canal therapy - a five-year retrospective study. Int Endod J 1993; 26: 321-333.

3. Friedman S. Etiological factors in endodontic post treatment disease: apical periodontitis associated with root filled teeth. Endod Topics 2003; 6: 1-2.

4. Wu M-K, Dummer P M H, Wesselink P R. Consequences of and strategies to deal with residual post treatment root canal infection. Int Endod J 2006: 39: 343-356.

5. Ørstavik D. Time-course and risk analysis of the development and healing of chronic apical periodontitis in man. Int Endod J 1996; 29: 150-155.
6. Huumonen $S, \emptyset$ rstavik D. Radiological aspects of apical periodontitis. Endod Topics 2002; 1: 3-25.

7. Ørstavik D, Kerekes K, Eriksen H M. The periapical index: a scoring system for radiographic assessment of apical periodontitis. Endod Dent Traumatol 1986; 2: 20-34.

8. Strindberg $L Z$. The dependence of the results of pulp therapy on certain factors. Acta Odontol Scand 1956; 14: supplement 21.

9. Molven 0, Halse A. Success rates for gutta-percha and kloroperka N-O root fillings made by undergraduate students: radiographic findings after 10-17 years. Int Endod J 1988: 21: 243-250.

10. FGDP (UK) Royal College of Surgeons of England. Selection Criteria for Dental Radiography. Good practice guidelines 2004. p68.
11. Matsumoto T, Nagai T, Kazuhiko I et al. Factors affecting successful prognosis of root canal treatment. J Endod 1987; 13: 239-242.

12. Pitt Ford TR, Ørstavik D. Essential endodontology: prevention and treatment of apical periodontitis. $p$ 371. Cornwall: Blackwell, 1998.

13. Byström A, Happonen R-P, Sjogren U, Sundqvist G Healing of periapical lesions of pulpless teeth after endodontic treatment with controlled asepsis. Endod Dent Traumatol 1987: 3: 58-63.

14. Friedman S. Consideration and concepts in management of post treatment endodontic disease - treatment failures. Endod Topics 2002; 1: 54-78.

15. Bergenholtz G, Horsted-Bindslev P, Reit C.

Textbook of endodontology. pp 204-205. Denmark: Blackwell Munksgaard, 2003. 